\title{
Prediction of Gas Leak Tightness of Superplastically Formed Products
}

\author{
Corijn H.C. Snippe ${ }^{a}$ and T. Meinders ${ }^{b}$ \\ ${ }^{a}$ National Institute for Subatomic Physics (Nikhef) \\ PO Box 41882, 1009 DB Amsterdam, The Netherlands \\ e-mail: csnippe@nikhef.nl,web page: http://www.nikhef.nl \\ ${ }^{b}$ University of Twente, Faculty of Engineering Technology \\ PO Box 217, 7500 AE Enschede, The Netherlands \\ e-mail:V.T.Meinders@ctw.utwente.nl,web page: http://www.tm.ctw.utwente.nl
}

\begin{abstract}
In some applications, in this case an aluminium box in a subatomic particle detector containing highly sensitive detecting devices, it is important that a formed sheet should show no gas leak from one side to the other. In order to prevent a trial-and-error procedure to make this leak tight box, a method is set up to predict if a formed sheet conforms to the maximum leak constraint. The technique of superplastic forming (SPF) is used in order to attain very high plastic strains before failure. Since only a few of these boxes are needed, this makes, this generally slow, process an attractive production method. To predict the gas leak of a superplastically formed aluminium sheet in an accurate way, finite element simulations are used in combination with a user-defined material model. This constitutive model couples the leak rate with the void volume fraction. This void volume fraction is then dependent on both the equivalent plastic strain and the applied hydrostatic pressure during the bulge process (backpressure).
\end{abstract}

Keywords: Gas leak, superplasticity, sheet forming simulations.

PACS: $62.20 . \mathrm{fq}$

\section{INTRODUCTION}

Superplastic forming is a process in which a sheet of superplastic material is pressed into a one-sided die by means of gas pressure. A superplastic material can attain very high plastic strains before failure, provided that the forming temperature is high and the maximum strain rate in the material is very low. The flow stress is highly strain rate dependent, which can be expressed in the strain rate sensitivity $m$. A theoretical background of the mechanics of superplastic material behavior is described in Section 1.

The material which is the subject of this research, is called ALNOVI-1, by Furukawa Sky Aluminium Ltd., and is a (nearly) superplastic aluminum based on AA 5083. The material constituents can be read in Table 1.

With this material, free bulge experiments have been carried out. Within these experiments, the forming pressure is a function of time and target strain rate. This means that the forming pressure during a whole experiment is such that a predefined target strain rate within the material will not be exceeded. The variables used in the experiments are the target

TABLE 1. Element constitution of ALNOVI-1.

\begin{tabular}{lcccccc}
\hline Element & $\mathbf{M g}$ & $\mathbf{M n}$ & $\mathbf{C r}$ & $\mathbf{F e}$ & $\mathbf{S i}$ & $\mathbf{A l}$ \\
\hline$[\%]$ & 4.5 & 0.7 & 0.12 & $<0.1$ & $<0.1$ & $\mathrm{Bal}$.
\end{tabular}


strain rate and the extra applied hydrostatic pressure during the tests. From the formed sheets, the bulge height, the top thickness and the leak tightness have been measured. The bulge experiments will be described in section 2 .

Uniaxial experiments, which had already been carried out, showed the one-dimensional behavior of ALNOVI-1. The mechanical one-dimensional behavior can be described in terms of an initial flow stress, which is dependent on the equivalent plastic strain rate. This part shows the strong dependency of the flow stress on the strain rate, so a high strain rate sensitivity had been measured. Secondly, strain hardening was present, which could be described in terms of a Voce type of hardening. Thirdly, a strain softening part in the model arises where the void volume fraction reaches a value such that it influences the mechanical behavior. These three parts of the model are briefly described in Section 3, this model is then used as a basis to carry out simulations of the bulge experiments, where the calculation of the leak tightness is an important issue.

Section 4 shows the comparison between the experimental results and the finite element calculations. Simulations have been done for the situation in which no backpressure is applied, and for the situation that a backpressure of 30 bar $\left(3.0 \mathrm{~N} / \mathrm{mm}^{2}\right)$ has been applied during the forming stage. Three target strain rates were used: 6,12 and $18 \cdot 10^{-4} \mathrm{~s}^{-1}$. The calculated leak values were compared with the experimental values, which is also presented in this section.

The last part of this article is dedicated to the conclusions and an outlook of the future work. This work includes mostly the development of a more enhanced material model.

\section{SUPERPLASTICITY}

Superplasticity can be defined as the ability of polycrystalline materials to exhibit very high elongations prior to failure. This high elongation (ranging from a few hundred to several thousand percent) is very strictly limited by a narrow range of operating temperature and strain rate. Within this range, superplastically deformed materials show a very high resistance against necking; the material gets thinner in a very uniform manner. Deformation should be carried out at an elevated temperature, which is generally higher than temperatures needed for conventional warm forming. The superplastic aluminum alloy ALNOVI-1 shows for instance an optimal superplastic temperature of $520^{\circ} \mathrm{C}$. Typical optimal strain rates for superplastic behavior range in the order of $10^{-4}$ to $10^{-2} \mathrm{~s}^{-1}$.

A requirement of a material to behave superplastically, is the fine grain size, which can vary from material to material between 1 and $10 \mu \mathrm{m}$. The grains should be randomly oriented in the material, causing it to behave isotropically, and may not grow during plastic deformation, in order to maintain the superplastic properties throughout the entire forming process. In the remainder of this section, the mechanical behavior of the material will be explained as a phenomenological model.

The flow stress shows a high strain rate dependency, as can be seen in Figure 1(a). Here, the initial flow stress, i.e. at an equivalent plastic strain of zero, is shown as a function of the equivalent plastic strain rate. In log-log scale, the curve can be described as one with a sigmoidal shape.

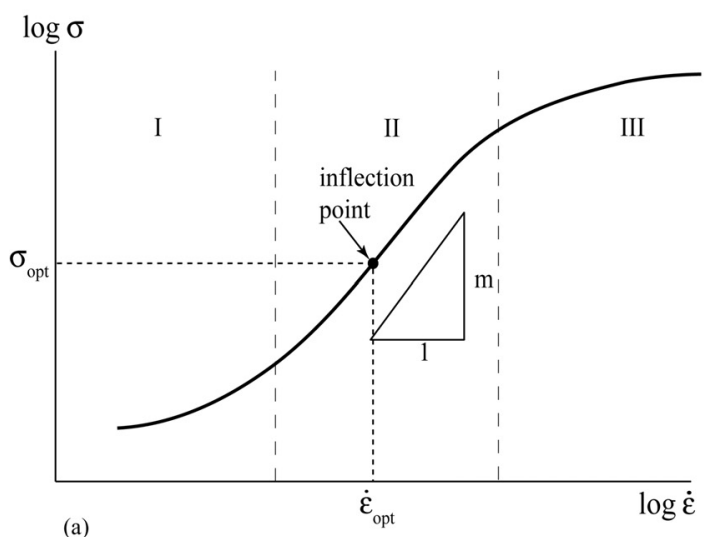

(a)

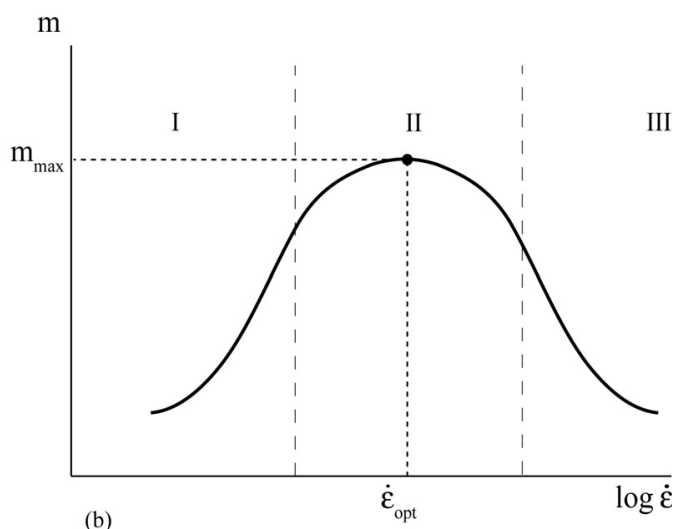

(b)

III
FIGURE 1. Typical superplastic material behavior. (a): Sigmoidal curve showing stress-strain behavior. (b): Strain rate sensitivity parameter $\mathrm{m}$ as function of the strain rate. 
Figure 1(b) shows the strain rate sensitivity as function of the equivalent plastic strain rate. The optimal strain rate for superplasticity to occur is at the top of the curve, where the strain rate sensitivity shows a peak.

Superplastic materials also show strain hardening, but this is generally less than for conventional plasticity observed in metals. Aluminum at elevated temperatures show a Voce type of strain hardening, which can be expressed as an asymptotic function with two material constants: the saturation stress $\Delta \sigma$ and a constant $\varepsilon_{0}$ which represents the speed at which the asymptote is approached:

$$
\sigma_{\mathrm{fh}}=\sigma_{\mathrm{f} 0}+\sigma_{\mathrm{h}}=\sigma_{\mathrm{f} 0}+\Delta \sigma \cdot\left(1-\exp \left(-\dot{\varepsilon}^{\mathrm{p}} \varepsilon^{\mathrm{p}} / \varepsilon_{0}\right)\right)
$$

where $\varepsilon^{p}$ is the equivalent plastic strain and $\sigma_{\mathrm{f} 0}$ is the initial flow stress. ALNOVI-1 also showed this kind of strain hardening as followed from the uniaxial experiments.

The initiation and growth of internal voids at high plastic strains are the cause of a strain softening effect. The void volume fraction $\xi$ can be acccounted for in the one-dimensional constitutive relation by means of a softening factor on the stress $\sigma_{\mathrm{fh}}$ which is determined by three material constants:

$$
\mu_{\mathrm{s}}=\left(1-\eta_{1} \xi^{\eta_{2}}\right)^{\eta_{3}}
$$

The void volume fraction is dependent on the equivalent plastic strain and for ALNOVI-1, the relation between these two measures is given in Figure 2. Note that the void volume fraction is hardly dependent on the equivalent plastic strain rate, so this quantity was not taken into account in the constitutive model for the calculation of the void volume fraction. The void volume fraction was implemented as a bilinear relationship between $\xi$ and $\varepsilon$.

These void volume fractions were measured in a situation where no hydrostatic pressure was applied, since this was not possible in the uniaxial experimental setup. It is known, however, that applying such a pressure can inhibit the formation and growth of voids. This will then postpone the softening effect on the flow stress, which means that the material can reach higher plastic strains until failure.

\section{BULGE EXPERIMENTS}

The purpose of the bulge experiments is to establish a relationship between the leak tightness of the formed sheet with respect to helium and the equivalent plastic strain (which is directly related to the void volume fraction, see Figure 2). Bulges were formed at three different speeds, which represent the maximum strain rate occurring in the sheet during the

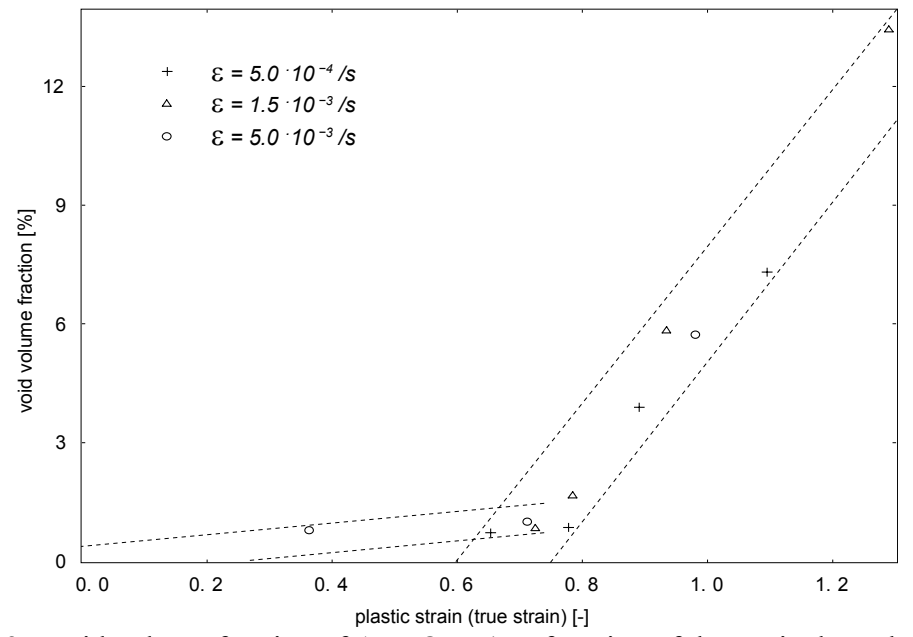

FIGURE 2. Void volume fraction of ALNOVI-1 as function of the equivalent plastic strain. 


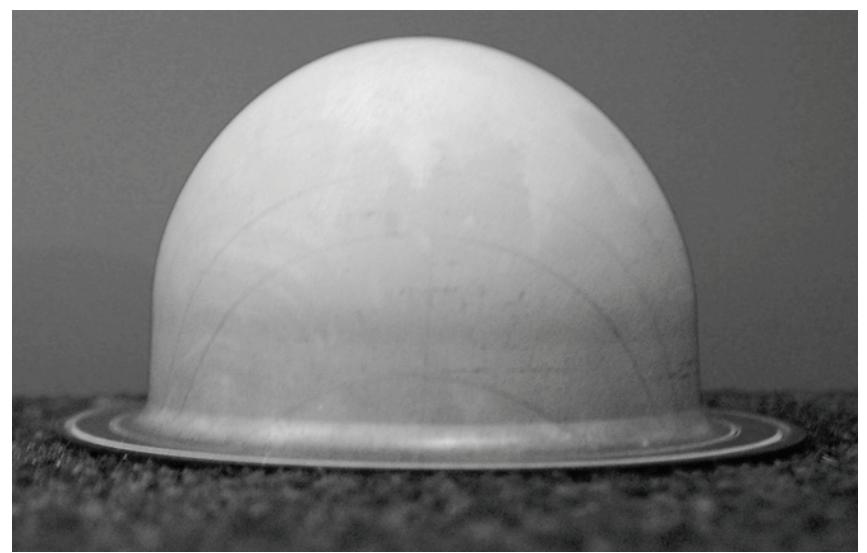

FIGURE 3. Example of a formed bulge, the bulge diameter is $70 \mathrm{~mm}$, the sheet diameter is $91.5 \mathrm{~mm}$. The initial sheet thickness is $0.8 \mathrm{~mm}$. This bulge reached a height of $51.3 \mathrm{~mm}$ and a top wall thickness of $0.15 \mathrm{~mm}$.

forming process. Another variable was the applied backpressure during forming. The third variable was the time at which a particular test was stopped. The initial thickness of the sheets was $0.8 \mathrm{~mm}$, the diameter was $91.5 \mathrm{~mm}$, the diameter of the formed bulges was $70 \mathrm{~mm}$, see Figure 3 for an example of a bulged sheet.

The leak tightness of a bulged sheet is measured by applying a 1 bar helium pressure on the inner side of the sheet and measuring the amount of helium which leaks through at the other side of the bulged sheet. The leak value is generally expressed as the amount of gas (i.e. a pressure times a volume) which passes through the sheet per time unit, leak measuring devices use the unit mbar. $1 / \mathrm{s}$.

Figure 4 shows the leak rate results of a set of formed bulges, in which the leak rate is plotted against the bulge height which was reached during the test. From this plot it is clear that at higher values for the applied backpressure (visible by the grey bands in the plot), leak values at the same bulge height are lower. Secondly, by applying a backpressure, higher bulges can be formed.

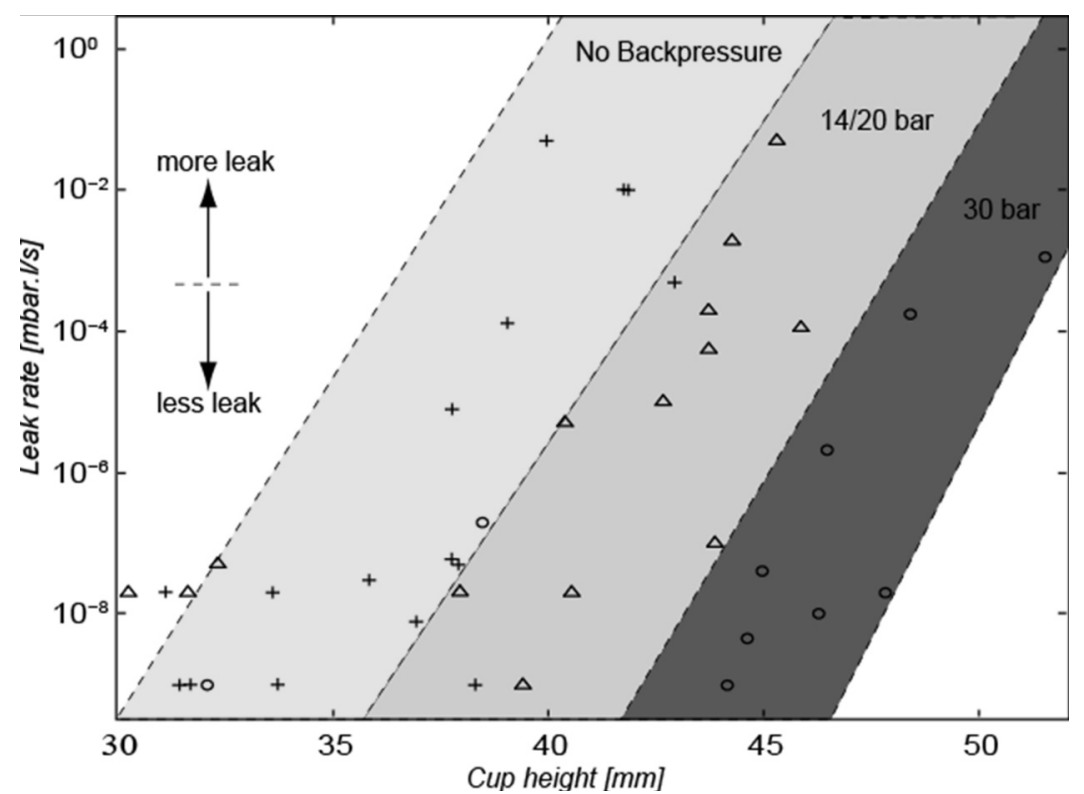

FIGURE 4. Plot showing the leak rate versus the reached cup height in the bulge test. The highest bulges can be reached at 30 bar backpressure, visible by the darkest grey band. 
All initial blanks were rasterized with a pencil-drawn grid, which made it possible to determine the in-plane plastic strains. Because in the top of the bulge an equibiaxial stress and strain state is present, the equivalent plastic strain is simply twice the measured one-dimensional strain on the top in case of von Mises plasticity. From the uniaxial experiments, it followed that the Lankford strain ratio $R$ is less than 1, the material behaves in-plane isotropic with $R=$ 0.825. Furthermore, it is known for aluminum at elevated temperatures that the more general, non-quadratic, Hosford yield criterion is more applicable, where $n=8$ in the following equation for the equivalent stress in principal directions:

$$
\bar{\sigma}=\left\{\frac{1}{R+1}\left(\sigma_{1}^{n}+\sigma_{2}^{n}\right)+\frac{R}{R+1}\left(\sigma_{1}-\sigma_{2}\right)^{n}\right\}^{1 / n}
$$

In an equibiaxial state, it follows from $\sigma_{1}=\sigma_{2}$ and $\varepsilon_{1}=\varepsilon_{2}$ and the equation

$$
\boldsymbol{\sigma}: \boldsymbol{\varepsilon}^{\mathbf{p}}=\bar{\sigma} \bar{\varepsilon}^{\mathrm{p}}
$$

that for $\mathrm{n}=8$ and $\mathrm{R}=0.825$ the equivalent plastic strain in the top is equal to $1.98 \varepsilon_{1}$, so this resembles much the von Mises situation. Besides global leak rate measurements, also local measurements have been carried out, in order to find out which part of the total leak traverses the top part of the bulge. These measurements were done with a helium sniffer with a $2 \mathrm{~mm}$ diameter measuring straw. These leak results resembled very much the global leak results (within 5\%), so the conclusion can be drawn that the major part of the gas leak is caused by a very small region in the top of the bulge. The measured leak rate as function of the equivalent plastic strain in the top is depicted in Figure 5.

\section{MATERIAL MODELLING}

In order to predict the leak rate of a superplastically formed product, the results of the uniaxial and biaxial experiments have been used to formulate a constitutive model to be used in finite element simulations. Part of this constitutive model has already been incorporated into a user-defined material model.

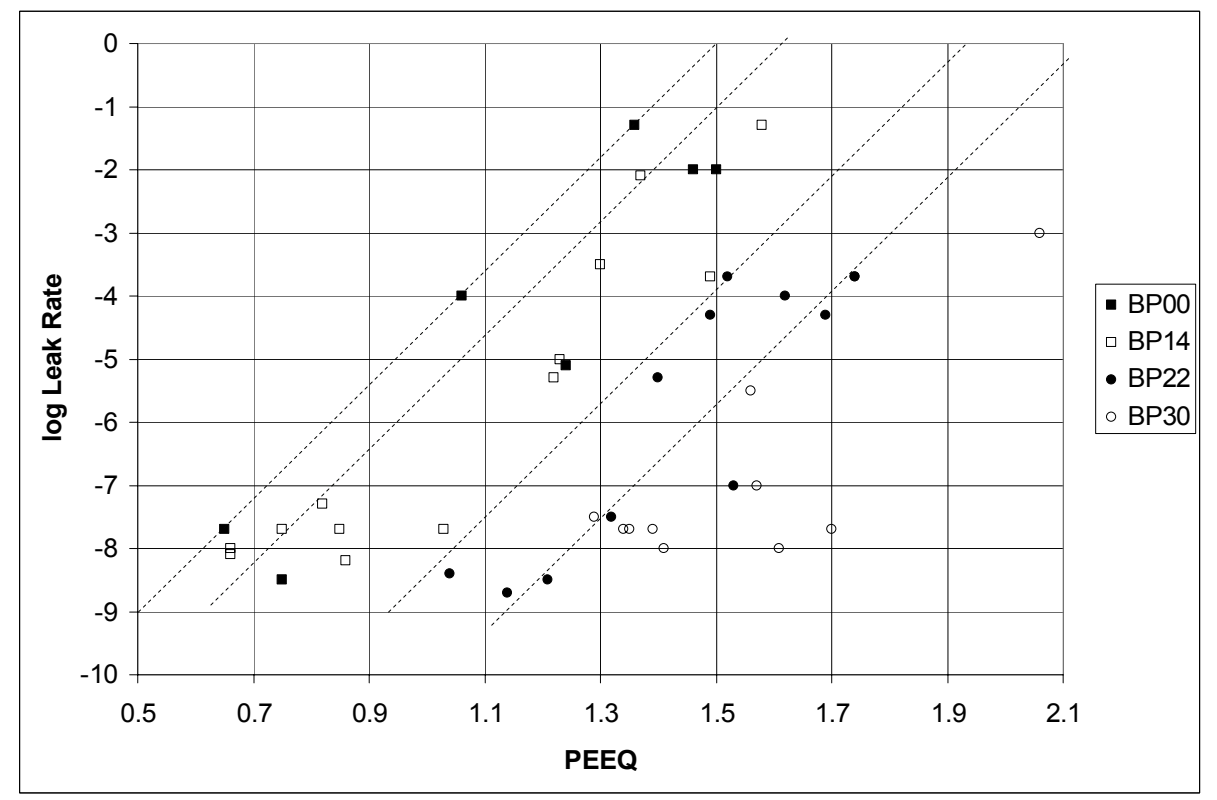

FIGURE 5. Plot showing the leak rate versus the equivalent plastic strain, for four values of the backpressure (BP). The four straight lines define the borders of the 'safe zone'. 
TABLE 2. Material parameters describing the phenomenological mechanical properties of ALNOVI-1.

\begin{tabular}{cccccccc}
\hline \multicolumn{2}{l}{ Initial flow } & \multicolumn{2}{c}{ Hardening } & \multicolumn{2}{c}{ Softening } & \multicolumn{2}{c}{ Void growth } \\
\hline $\mathrm{a}$ & 1.1274 & $\Delta \sigma$ & $3.31 \mathrm{E} 6$ & $\eta_{1}$ & 5.24 & $\mathrm{c} 1$ & 0.015 \\
$\mathrm{~b}$ & $2.2207 \mathrm{E}-4$ & $\varepsilon_{0}$ & $1.2 \mathrm{E}-4$ & $\eta_{2}$ & 0.946 & $\mathrm{c} 2$ & 0.141 \\
$\mathrm{c}$ & -2.901 & & & $\eta_{3}$ & 1.272 & $\mathrm{c} 3$ & 0.021 \\
$\mathrm{~d}$ & 6.2025 & & & & & $\varepsilon_{\mathrm{c}}$ & 0.74
\end{tabular}

The uniaxial experiments have led to a set of material constants which describe the initial flow stress, the Voce strain hardening part and the softening part due to the void volume fraction. The latter two are described in Equations (1) and (2), an equation to describe the initial flow stress is based on the equation for a sigmoidal curve and reads

$$
\log \left(\sigma_{f 0}\right)=\frac{1}{a+b \exp (c \log (\dot{\varepsilon}))}+d
$$

in which $a, b, c$ and $d$ are material constants. For the Voce hardening part, two material constants were determined, namely $\Delta \sigma$ and $\varepsilon_{0}$. The part that describes the strain softening as function of the void volume fraction is described by the three material constants $\eta_{1}, \eta_{2}$ and $\eta_{3}$. The void volume fraction $\xi$ has been measured in the uniaxial experiments as only dependent on the equivalent plastic strain rate, and is described by three material constants: $c_{1}$ and $c_{2}$ describe the individual slopes, as can be seen in Figure 2, a third constant, $\varepsilon_{\mathrm{c}}$, is the critical strain at which the slope changes.

The influence of the backpressure could not be measured by means of the uniaxial experiments, only by means of the bulge experiments. Since the application of a backpressure slows down the nucleation and growth of voids, it is therefore believed that the backpressure only influences the softening part of the uniaxial constitutive model. An extra parameter $c_{3}$ determines a shift of the critical plastic strain $\varepsilon_{\mathrm{c}}$ and both the slopes $c_{1}$ and $c_{2}$. These three constants change with the backpressure according to

$$
\begin{gathered}
c_{1,2}^{*}=\frac{c_{1,2}}{1+c_{3} p_{h}} \\
\varepsilon_{\mathrm{c}}^{*}=\varepsilon_{\mathrm{c}}\left(1+c_{3} p_{\mathrm{h}}\right)
\end{gathered}
$$

Values of the parameters described here are summarized in Table 2.

Incorporating the leak into the model is somewhat more complicated, since the leak measurements show some variation with the equivalent plastic strain. Therefore it is difficult to obtain a one-to-one relationship between these two measurements. The drawn straight lines in Figure 5 show the borders of a so-called 'safe zone', where it is assumed that if a value for the equivalent plastic strain is calculated, the leak rate will never lie on the left hand side of the corresponding 'safe zone' line. These lines are used for calculating the leak rate as function of the equivalent plastic strain. Since it is known that the major part of the gas leak will traverse through the top of the bulge, the maximum equivalent plastic strain from the simulations will be used in the equations between leak and strain.

\section{SIMULATIONS}

The uniaxial constitutive model as explained in the previous section is implemented into an ABAQUS user-defined material model. The results shown in this section concern the case where von Mises flow is present and the material is fully isotropic. Figure 6 shows the results in terms of equivalent plastic strain of two simulations, both at a target strain rate of $6 \cdot 10^{-4} \mathrm{~s}^{-1}$. The picture on the left represents the bulge forming without the application of a backpressure, whereas the picture on the right are the simulation results where a 30 bar backpressure has been applied during the forming stage. 


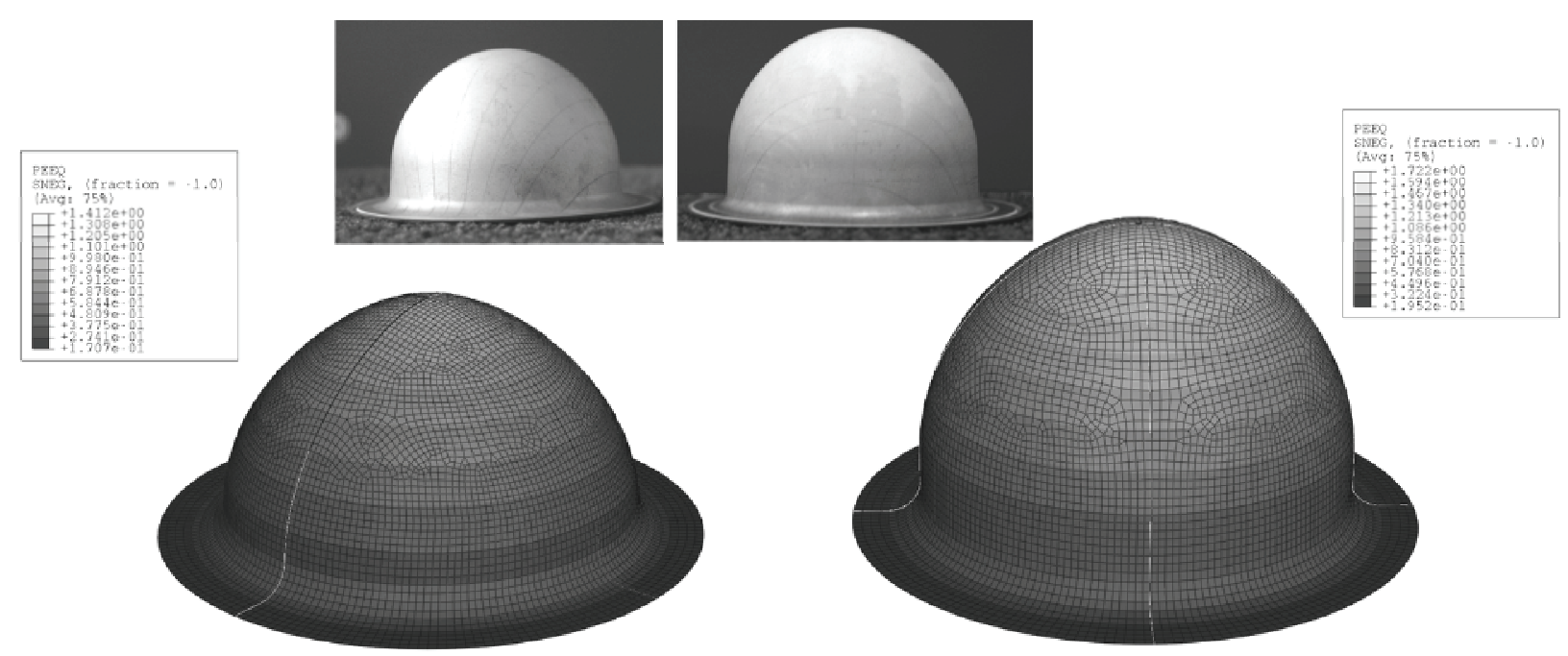

FIGURE 6. Simulated bulge experiments, left picture shows a simulation at a target strain rate of $6 \cdot 10^{-4} \mathrm{~s}^{-1}$ without the application of a backpressure; the picture on the right shows the simulation at the same target strain rate, but with an application of 30 bar backpresure. On top: photos of the corresponding bulges from the experiments.

The equivalent plastic strain as simulated and as calculated from the experiments show a quite good similarity if this measure is plotted against the bulge height, the calculated equivalent plastic strains are somewhat overestimated. This is done in Figure 7 (left) for the case of a bulge formed at the three target strain rates of $6 \cdot 10^{-4}, 12 \cdot 10^{-4}$ and $18 \cdot 10^{-4} \mathrm{~s}^{-1}$ and a backpressure of 30 bar. This similarity is less if the equivalent plastic strain is plotted against the forming time, as shown in Figure 7 (right).

From Figure 7 on the left it can be concluded that if the simulated equivalent plastic strain in the top part of the bulge resembles the experimental values, the strain distribution in the bulge can be considered an acceptable approximation. This approximation could be further improved a Hosford yield criterion instead of the standard von Mises yield criterion would be applied. The figure on the right shows that at a low target strain rate, the deviation from the experiment is quite high. This is caused by the fact that at higher strains, softening causes instability effects, leading to larger deviations. This does not necessarily mean that the uniaxial constitutive model at high strains is inadequate.

Another check for the accuracy of the results is the top thickness. Table 3 gives the top thickness results for the highest bulges which were reached for the three target strain rates and the two backpressure values, both for the
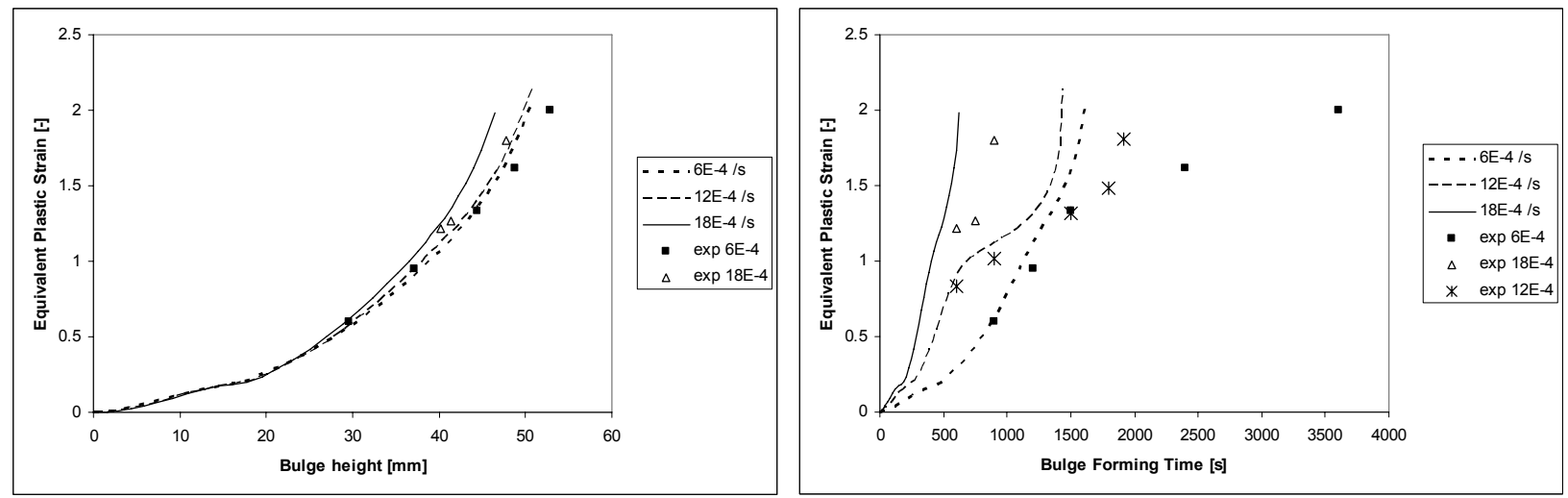

FIGURE 7. Comparison of the simulated equivalent plastic strain rate versus the bulge height (left), and of the simulated equivalent plastic strain versus the bulge forming time (right). The marks in the figures represent experimental values. 
TABLE 3. Top thickness results from the bulge experiments and the FE simulations. Note that the initial sheet thickness is $0.8 \mathrm{~mm}$.

\begin{tabular}{|c|c|c|c|c|c|c|}
\hline Target strain rate $\left[\mathrm{s}^{-1}\right]$ & \multicolumn{2}{|c|}{$6 \cdot 10^{-4}$} & \multicolumn{2}{|c|}{$12 \cdot 10^{-4}$} & \multicolumn{2}{|c|}{$18 \cdot 10^{-4}$} \\
\hline Backpressure [bar] & $\mathbf{0}$ & 30 & $\mathbf{0}$ & 30 & $\mathbf{0}$ & 30 \\
\hline Thickness, exp [mm] & 0.24 & 0.16 & 0.24 & 0.18 & 0.24 & 0.15 \\
\hline Thickness, sim [mm] & 0.16 & 0.14 & 0.20 & 0.16 & 0.18 & 0.13 \\
\hline
\end{tabular}

experiments and the simulations. The thickness results for the simulations without an applied backpressure shows an underestimation, this is caused by the already mentioned instabilities during softening. The thickness results with an application of 30 bar backpressure show a better resemblance, but there is still a small amount of structural underestimation. This is caused by the fact that the the simulations presume volume preservation, whereas this is not the case because of void growth.

\section{CONCLUSION / OUTLOOK}

The superplastic forming can be simulated by means of the finite element method by applying a uniaxial material model in which three parts are represented: firstly the initial flow stress, which is highly dependent on the strain rate.; secondly, a hardening part, which can best be described with a Voce hardening model, as is convenient for aluminum at elevated temperatures; thirdly, as oftening part, caused by the initiation and growth of internal voids. When using this model for bulge forming simulations, where the standard von Mises flow criterion is used in metal plasticity, deviations occur between the experimental and simulation results, the equivalent plastic strain is overestimated a small amount. Application of the Hosford flow criterion may overcome this problem.

The effect of a hydrostatic pressure during the forming process which was seen in the experiments, can also be accounted for. This does not mean that a pressure-dependent material model needs to be coded, because it is known that during the forming process, the hydrostatic pressure does not change.

The next step in the material modelling will therefore be the implementation of this Hosford criterion, in which also the out-of-plane anisotropy is defined, by means of the Lankford strain ratio. Also, the resulting thickness needs to be corrected, because the occurrence of internal voids does not imply a volume preservation of the macroscopic material, which is generally assumed in metal plasticity calculations. The finite element program ABAQUS contains an extended Gurson metal plasticity material model, but this seemed to be inadequate for superplastic bulge forming simulations, void volume fractions could not be predicted very well.

\section{REFERENCES}

[1] T. Naka, Y. Nakayama, T. Uemori, R. Hino, and F. Yoshida. Effects of temperature on yield locus for 5083 aluminium alloy sheet. Journal of Materials Processing Technology, 140:494-9, 2003.

[2] R.A. Vasin, F.U. Enikeev, M.I. Mazurski, and O.S.Munirova. Mechanical modelling of the universal superplastic curve. Journal of Materials Science, 35:2455-66, 2000.

[3] D. Banabic, T. Kuwabara, T. Balan, D.S. Comsa and D. Julean. Non-quadratic yield criterion for orthotropic sheet metals under plane-stress conditions. International Journal of Mechanical Sciences, 45:797-811, 2003.

[4] Roger W. Logan. Finite-Element Analysis of Earing Using Non-Quadratic Yield Surfaces. NUMIFORM '95, Cornell University, Ithaca NY

[5] M. Khaleel, H. Zbib, and Nyberg E. Constitutive modeling of deformation and damage in superplastic materials, International. Journal. of Plasticity, 17:277-296, 2001.

[6] G.Y. Li, M.J. Tan, and K.M. Liew. Three-dimensional modeling and simulation of superplastic forming. Journal of Materials Processing Technology, 150: 76-83, 2004 